\title{
Classification of Hong Kong Prevailing Standard Skies
}

\author{
D.H.W. Li* and H.L. Tang
}

\author{
Building Energy Research Group, Department of Building and Construction, City University of Hong Kong, Hong Kong \\ SAR, China
}

\begin{abstract}
In 2003, the International Commission on Illumination (CIE) adopted a range of 15 standard skies covering the whole probable spectrum of usual skies in the world. A good understanding of the sky luminance distribution is essential to daylighting developments and energy-efficient building designs. This paper studies the work on the identification of Hong Kong prevailing standard skies. Ten-minute data recorded between 1999 and 2005 in Hong Kong were used for the analysis. An approach to identify a subset of 6 sky standards including overcast, partly cloudy and clear sky types was proposed. The performance of the proposed approach was evaluated against the measured data in terms of root-meansquare error (RMSE). The statistical analysis showed that the proposed method produced acceptable agreements with the measured data and the overall RMSE was computed of $30 \%$
\end{abstract}

\section{INTRODUCTION}

Sky luminance distributions are essential to architects, engineers and scientists for building designs, daylight-linked lighting control studies, and climatology and pollution analyses. However, the sky luminance distributions are seldom available. Using experimental data, a number of empirical models have been established to investigate the characteristics of sky patterns under various sky conditions [1-3]. Recently, Kittler et al. [4] have proposed a range of 15 standard skies based on a detailed analysis of selected luminance scans measured in Berkeley $\left(37.6^{\circ} \mathrm{N}, 122.4^{\circ} \mathrm{W}\right)$ during $1985-$ 86 , supplemented by data from scans recorded in Tokyo $\left(35.8^{\circ} \mathrm{N}\right.$ and $\left.139.8^{\circ} \mathrm{E}\right)$ and Sydney $\left(33.9^{\circ} \mathrm{S}\right.$ and $\left.151.2^{\circ} \mathrm{E}\right)$ during 1992. The set of 15 standard skies adopts the existing International Commission on Illumination (CIE) standard overcast and clear skies covering the whole probable spectrum of skies in the world. In 2003, these 15 sky distributions were adopted by the CIE as the standard skies [5].

The sky luminance distributions are caused by various factors including the solar position, the atmospheric turbidity and the air pollution, and the cloud amount, type and pattern that can affect unpredictably sunlight and skylight [6]. Sky conditions of the same category would have similar sky luminance distributions and the corresponding climatic parameters and indices would be within certain ranges. Such analyses can help the identification of sky patterns and complicated mathematical expressions to model the sky distributions are not required. Once the skies have been known, the basic daylight illuminance at any surfaces and some important meteorological elements can be obtained for subsequent investigations. The essential issue would be the frequency of occurrence for the individual sky standards appearing in a given location [7]. Many researchers proved that the $15 \mathrm{CIE}$ standard skies provide a good overall framework for representing the actual sky conditions and a subset of three to five

Building Energy Research Group, Department of Building and Construction, City University of Hong Kong, Hong Kong SAR, China;

E-mail: bcdanny@cityu.edu.hk luminance distributions were sufficient to describe the sky conditions at a particular region [8-10]. As suggested by Kittler et al. [4], the ratio of zenith luminance to horizontal diffuse illuminance $\left(\mathrm{L}_{\mathrm{z}} / \mathrm{D}_{\mathrm{v}}\right)$ can characterize the momentary sky brightness and theoretically can identify the 15 standard sky patterns. However, the $\mathrm{L}_{\mathrm{z}} / \mathrm{D}_{\mathrm{v}}$ theoretical curves for the 15 standard skies are not parallel and they intersect each other at solar altitude of $35^{\circ}$ or more. Using $\mathrm{L}_{\mathrm{z}} / \mathrm{D}_{\mathrm{v}}$ for a place where high solar altitude dominates can lead to ambiguous results [11]. Bartzokas et al. $[12,13]$ modified the $\mathrm{L}_{\mathrm{z}} / \mathrm{D}_{\mathrm{v}}$ sky classification method to study the daylight climate at two cities in Central Europe but a certain amount of luminance data were excluded for analysis. There are a number of climatic parameters appropriate to identify the daylight climates [14]. This paper presents the work on the classification of prevailing sky luminance distributions for Hong Kong using some appropriate climatic parameters. A comparative assessment of the best-fitting and the proposed methods against sky luminance data scanned between 1999 and 2005 is reported. Characteristics of the findings and implications for daylighting designs are discussed.

\section{HONG KONG CLIMATE \& SKY LUMINANCE MEASUREMENT}

Hong Kong has a total area of about $1000 \mathrm{~km}^{2}$ and is situated along the southern coast of China within the subtropical region, with latitude and longitude of $22.3^{\circ} \mathrm{N}$ and $114.2^{\circ} \mathrm{E}$, respectively. It has distinct seasonal changes in its weather due to its location on the south-east coast of the Asiatic continent opposite a vast expanse of the ocean. Winter months are between December and February. Mean temperature is around $15-18^{\circ} \mathrm{C}$ with the monsoon coming from the north and north-east. Spring is from March till early May, and is usually cloudy with periods of light rain. The summer season spans from late May till September. The monsoon wind blows from the south and south-east with an average temperature of $27-29^{\circ} \mathrm{C}$. It is hot and humid with occasional showers and thunderstorms. At times, typhoons strike Hong Kong and bring heavy rain and strong winds. Autumn is short and normally runs from October to November. Sunny 
bright skies dominate with dry conditions and a mean temperature of $25^{\circ} \mathrm{C}$.

The sky luminance distribution is measured by means of a sky scanner (EKO MS 300LR), which was manufactured and calibrated by the Japanese company EKO. It measures the luminance at 145 points (shown in Fig. 1) of the sky by scanning the sky domes. The celestial hemisphere splits into 145 angular patches in bands of constant elevation in a threeway symmetry about the zenith, giving the appropriate subdivision. The important parts of the sky scanner are housed in a weatherproof casing allowing continuous outdoor operation. Output data from the scanner are recorded on a microcomputer located inside the laboratory on the top floor. A VISUAL BASIC computer program is used to capture and transmit the measured data. To safeguard the sensor, the scanner does not record luminance data greater than 35 $\mathrm{kcd} / \mathrm{m}^{2}$ (points which are very close to the sun) by using an automatic shutter. Each scanning time is about 4 minutes and measurements are taken every 10 minutes.

\section{DATA QUALITY CONTROLS}

It should be noted that there are several causes affecting the accuracy of the measured sky luminance data. First, the celestial hemisphere was split into 145 circular angular sky patches for the luminance measurement. Such an arrangement, however, led to uncovered regions of the sky. Second, the measured data were based on discrete results rather than continuous analytical functions. Sky luminance between adjoining measurement points may have varied significantly. Third, the scanning time was about 4-minute and the measurement interval was 10-minute. Substantial variations in sky luminance may have occurred between each record. Also, for "out of range" measurements (points close to the solar position under non-overcast skies), an estimation of the sky luminance was made from a simple average of the luminance at nearby points and such conversion could introduce data distortion [15]. To eliminate spurious data and inaccurate

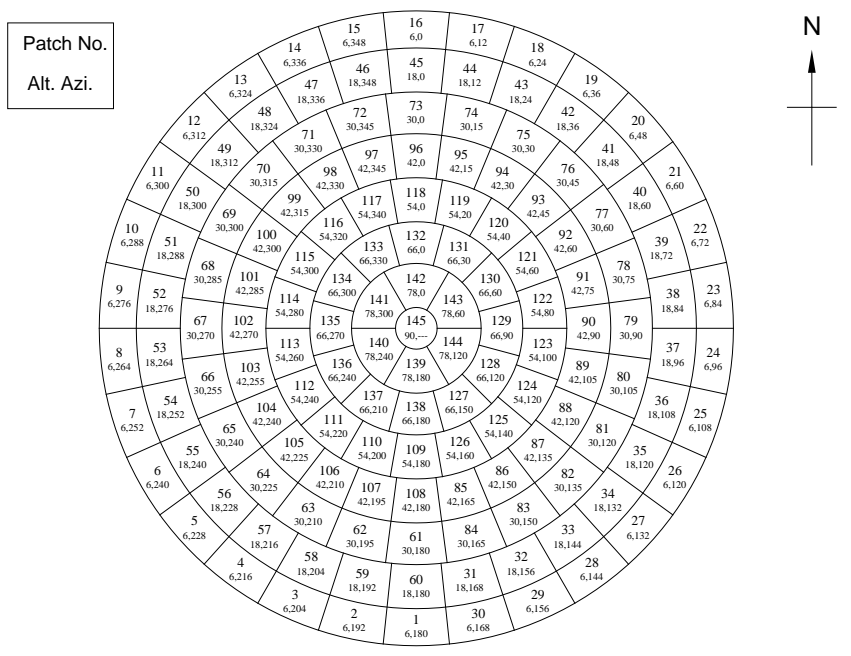

Fig. (1). The sequence of the 145 measurement points for sky scanning (Each patch shows its number together with the solar altitude and azimuth). measurements, some quality-control tests were adopted as follows:

Applying a shadow-ring correction to the measured horizontal diffuse data using the method described by LeBaron et al. [16].

1 Removing the sky scan data centered within $11^{\circ}$ of the sun's position.

2 Rejecting all diffuse data greater than the corresponding global values.

3 Rejecting all global data greater than the corresponding extraterrestrial solar components.

4 Rejecting all data measured at solar altitude $\left(\alpha_{\mathrm{s}}\right)$ less than $5^{\circ}$.

5 Rejecting all data with horizontal global values less than $20 \mathrm{~W} / \mathrm{m}^{2}$.

6 Rejecting all data with the direct-normal values [i.e. (global-diffuse)/sin $\alpha_{s}$ ] greater than the corresponding extraterrestrial solar component.

7 Rejecting all diffuse data greater than half of the corresponding extraterrestrial solar components (because of the improper shadow-ring adjustment).

8 Rejecting all sky luminance data when the difference between the corrected horizontal diffuse illuminance and the corresponding integrated horizontal diffuse illuminance based on the 145 scanned sky luminance points was greater than $30 \%$.

The measured data recorded from January 1999 to December 2005 were collected for the analysis. It is inevitable that there were some periods of missing data for various reasons, including instrumentation malfunction, power failure and sensor calibration. Considerable efforts were made to obtain a continuous record of data. After the quality-control test 68000 ten-minute readings were retained for analysis.

\section{CIE STANDARD SKIES}

The set of the 15 standard skies includes the CIE clear sky distributions, a uniform luminance distribution, and a close approximation to the CIE overcast sky. In general, the standard skies contain 5 clear, 5 intermediate and 5 overcast sky types covering the whole probable spectrum of skies in the world [17]. The distributions are described by continuous mathematical expressions that change smoothly in luminance from the horizon to the zenith and with the angular distance from the sun. The standard formula defining the relative luminance distribution on any standard sky can be considered as a combination of the gradation $\varphi(Z)$ and the indicatrix function $f(\chi)$ :

$$
\frac{\mathrm{L}}{\mathrm{L}_{\mathrm{Z}}}=\frac{f(\chi) \varphi(\mathrm{Z})}{f\left(\mathrm{Z}_{\mathrm{S}}\right) \varphi\left(0^{\circ}\right)} \cdots
$$

where $\mathrm{L}$ is the sky luminance in an arbitrary sky element $\left(\mathrm{kcd} / \mathrm{m}^{2}\right), \mathrm{L}_{\mathrm{z}}$ is the sky luminance at the zenith $\left(\mathrm{kcd} / \mathrm{m}^{2}\right), \mathrm{Z}$ is the zenith angle of a sky element ( $\mathrm{rad}), Z_{\mathrm{s}}$ is the zenith angle of the sun (rad) and $\chi$ is the scattering angle, the shortest angular distance between the sun and a sky element (rad). The standardized gradation is defined by appropriate $a$ and $b$ variables as: 
$\frac{\varphi(\mathrm{Z})}{\varphi\left(0^{\circ}\right)}=\frac{1+\mathrm{a} \exp (\mathrm{b} / \cos \mathrm{Z})}{1+\mathrm{a} \exp \mathrm{b}} \cdots$

The relative scattering indicatrix function can be modeled by an exponential function with adjustable coefficients $\mathrm{c}, \mathrm{d}$ and e as:

$\frac{f(\chi)}{f\left(\mathrm{Z}_{\mathrm{S}}\right)}=\frac{1+\mathrm{c}[\exp (\mathrm{d} \chi)-\exp (\mathrm{d} \pi / 2)]+\mathrm{e} \cos ^{2} \chi}{1+\mathrm{c}\left[\exp \left(\mathrm{d} \mathrm{Z}_{\mathrm{S}}\right)-\exp (\mathrm{d} \pi / 2)\right]+\mathrm{e} \cos ^{2} \mathrm{Z}_{\mathrm{S}}} \cdots$

The exponential term $\exp (\mathrm{d} \chi)$ represents the effect of Mie scattering, which decreases rapidly with distance from the sun. The $\cos ^{2} \chi$ term is due to the Rayleigh scattering and is zero at $90^{\circ}$ to the sun [18]. Both gradation and indicatrix functions are of 6 types covering the usual range of homogeneous cases from heavy overcast to cloudless skies. The combinations can form a large number of skies but only 15 relevant types were selected to be the standard set. (Table 1) shows the details of the 15 standard skies [19].

\section{RESULTS ANALYSIS}

To identify the set of standard skies in detail, sky luminance data were computed using the 15 sky standards and compared with the scanned sky luminance readings of the same period. The modeled sky luminance is normalised to the horizontal diffuse illuminance by multiplying all the luminance values with the normalisation ratio (NR) as:

$\mathrm{NR}=\frac{\Sigma \mathrm{L}_{\text {mea }} \cos \theta \sin \theta \mathrm{d} \theta \mathrm{d} \beta}{\Sigma \mathrm{l}_{\text {pred }} \cos \theta \sin \theta \mathrm{d} \theta \mathrm{d} \beta} \cdots$

where $\mathrm{L}_{\text {mea }}$ is the measured sky point luminance $\left(\mathrm{kcd} / \mathrm{m}^{2}\right)$, $1_{\text {pred }}$ is the predicted sky point luminance in relative form (dimensionless), $\theta$ is the altitude of a sky patch (rad) and $\beta$ is the azimuth of a sky patch (rad).

\section{(a). The Best-Fit Method}

The relative (not absolute value) sky luminance distribution for each sky patch was determined. Once the integrated diffuse illuminance or luminance at each sky point has been obtained, the absolute luminance values of all sky patches can be easily computed. An alternative would be to divide all sky luminance readings by the zenith luminance, but this can cause huge measuring error when the sun is near to the zenith [20]. For low latitude climates (e.g. Hong Kong) when the sun is frequently within a small angular distance from zenith, a normalization with respect to the diffuse horizontal illuminance would, therefore, be more appropriate.

Once normalized, the performance of each standard sky luminance model was assessed by the root-mean-square error (RMSE), which is obtained by subtracting the measured sky patch luminances from the modeled luminances of the 15 CIE standard skies, adding together the squares of these values, and dividing the total by the number of sky patches, and then taking the square root. The standard sky selected is the one with the lowest RMSE. (Fig. 2) shows (i) the frequency of occurrence of the 15 standard skies using the best-fitting approach, and (ii) the corresponding RMSE based on the complete standard set. Large variations can be observed for individual sky types and a detailed analysis of (Fig. 2) revealed that the overcast and clear skies (i.e. overcast - sky nos. 1 to 5 and clear - sky nos. 11 to 15) represent about $78 \%$ of the Hong Kong sky conditions. The intermediate skies (i.e. sky nos. 6-10) account for the remaining $22 \%$. The RMSE for all the 15 standard skies is $23.8 \%$.

\section{(b). The Proposed Procedure}

Luminance data for the whole sky are far less available. Climatic variables that are widely obtainable would be more

Table 1. Descriptions and Adjustable Coefficients of the 15 Standard Sky Types

\begin{tabular}{|c|c|c|c|c|c|c|}
\hline \multirow[b]{2}{*}{ No. } & \multirow[b]{2}{*}{ Type of sky } & \multicolumn{2}{|c|}{ for Gradation } & \multicolumn{3}{|c|}{ for Indicatrix } \\
\hline & & a & $\mathrm{b}$ & $\mathrm{c}$ & $\mathrm{d}$ & e \\
\hline 2 & Overcast with a steep gradation and slight brightening towards sun & 4 & -0.7 & 2 & -1.5 & 0.15 \\
\hline 3 & Overcast moderately gradated, azimuthal uniformity & 1.1 & -0.8 & 0 & -1 & 0 \\
\hline 5 & Overcast or cloudy with overall uniformity & 0 & -1 & 0 & -1 & 0 \\
\hline 6 & Partly cloudy with a uniform gradation and slightly brightening towards sun & 0 & -1 & 2 & -1.5 & 0.15 \\
\hline 7 & Partly cloudy with a brighter circumsolar effect and uniform gradation & 0 & -1 & 5 & -2.5 & 0.3 \\
\hline 8 & Partly cloudy, rather uniform with a clear solar corona & 0 & -1 & 10 & -3 & 0.45 \\
\hline 12 & Very clear/unturbid with a clear solar corona & -1 & -0.32 & 10 & -3 & 0.45 \\
\hline 13 & Cloudless polluted with a broader solar corona & -1 & -0.32 & 16 & -3 & 0.3 \\
\hline 14 & Cloudless turbid with a broad solar corona & -1 & -0.15 & 16 & -3 & 0.3 \\
\hline 15 & White-blue sky, turbid with a wide solar corona effect & -1 & -0.15 & 24 & -2.8 & 0.15 \\
\hline
\end{tabular}



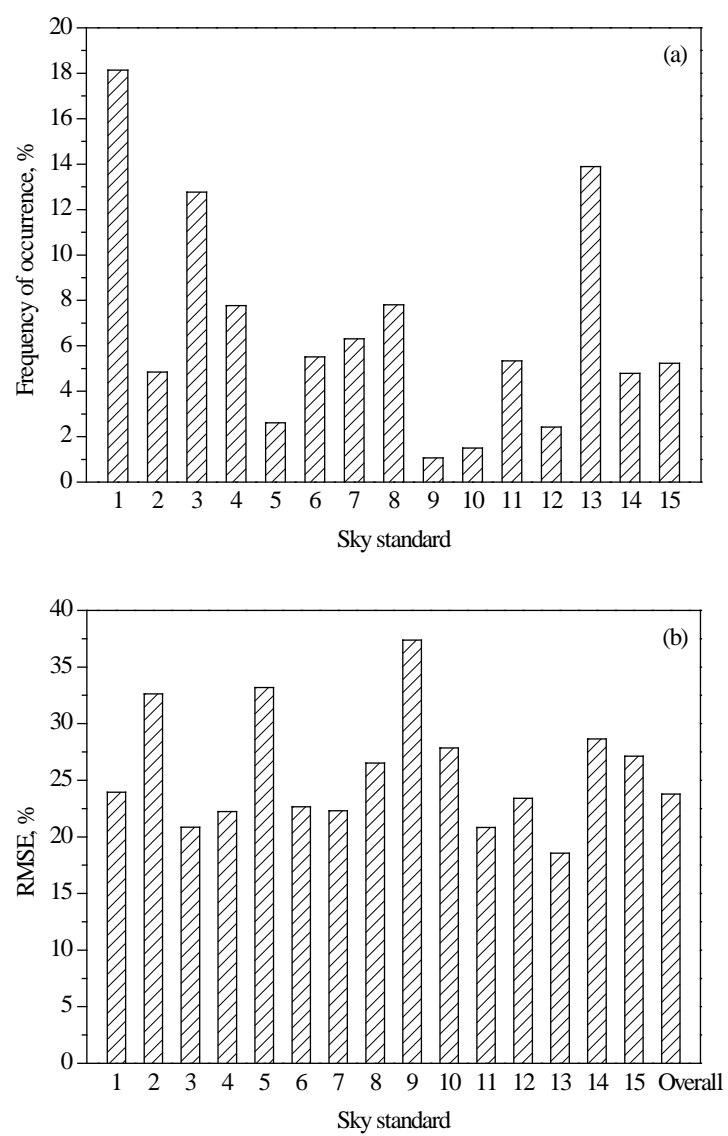

Fig. (2). (a) Frequency of occurrence of the 15 standard skies, (b) RMSE of the luminances of the actual skies relative to the 15 standard distributions in Hong Kong.

appropriate to classify sky conditions. It can be observed from (Fig. 2) that the best-fitting standard skies with high frequency of occurrence values give in low RMSE results and vice-versa. For example, sky types 2,5 and 9 have frequency of occurrence of less than $5 \%$ with the corresponding RMSEs of over $32 \%$. With the frequency of occurrence over $12.7 \%$, the RMSEs for sky nos. 1,3 and 13 are less than $24 \%$. This indicates that some standard skies appear infrequently and their exclusion can simplify the analysis without significantly lowering the overall accuracy. In general, a subset of the CIE standard skies including overcast, partly cloudy and clear sky conditions are sufficient to describe the daylight climates at any particular site [8,21]. Individual standard skies with high frequency of occurrence and low RMSE are appropriate to represent the prevailing sky condi-

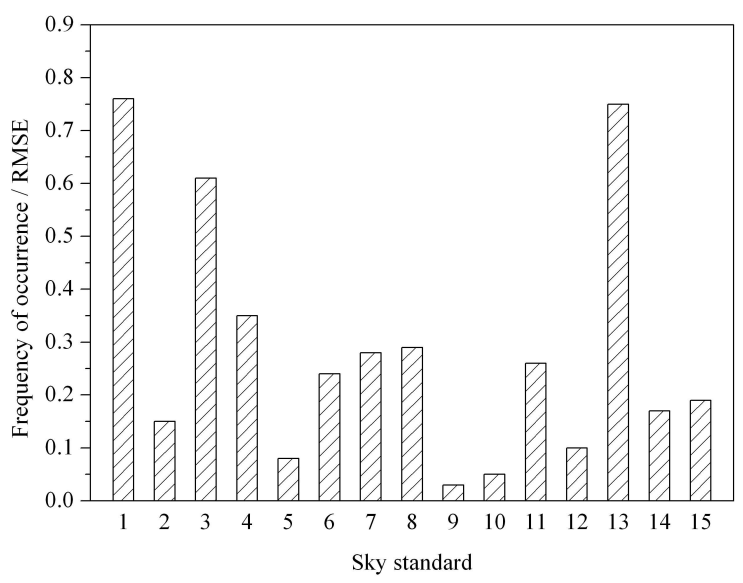

Fig. (3). The ratios of frequency of occurrence to RMSE for the 15 best-fitting sky standards.

tions for a given location. In this regard, the ratio of frequency of occurrence to RMSE for the 15 best-fitting standard skies was considered and (Fig. 3) presents the results. It can be seen that sky types 1 and 3 of overcast conditions have ratios over 0.6 , which is far more than the other 3 overcast sky types (i.e. sky nos. 2, 4 and 5). For partly cloudy conditions, the ratio for sky nos. 6,7 and 8 are between 0.24 and 0.3 but those for sky nos. 9 and 10 is close to zero. Referring to clear sky type, sky no. 13 has the highest ratio of over 0.75 . Based on these findings, sky nos. $1,3,6,7,8$ and 13 were selected to represent the prevailing sky standards in Hong Kong.

As proposed by Kittler and Darula [14], the pair $\mathrm{L}_{\mathrm{z}} / \mathrm{D}_{\mathrm{v}}$ and the ratio of horizontal global illuminance to the horizontal extraterrestrial illuminance $\left(G_{v} / E_{v}\right)$ is an effective hybrid sky-parameter to interpret the three typical sky conditions (i.e. overcast, partly cloudy and clear). When the atmosphere is clear, a small fraction of daylight illuminance is scattered, resulting in a predominant direct component with a large amount of $G_{v} / E_{v}$. For an overcast day, a large amount of outdoor illuminance is scattered, indicating a high portion of diffuse component with a lower $\mathrm{G}_{\mathrm{v}} / \mathrm{E}_{\mathrm{v}}$ reading. There are no clear-cut values for $L_{\mathrm{z}} / \mathrm{D}_{\mathrm{v}}-\mathrm{G}_{\mathrm{v}} / \mathrm{E}_{\mathrm{v}}$ to represent various typical sky conditions. Based on the measured Hong Kong data, we proposed the $L_{z} / D_{v}-G_{v} / E_{v}$ ranges for identification of the 3 typical skies [22]. (Table 2) summarizes the criteria adopted for the analysis. To distinguish between sky nos. 1 and 3 under the overcast dataset, the ratio $\mathrm{L}_{\mathrm{z}} / \mathrm{D}_{\mathrm{v}}$ was employed again. Darula and Kittler [23] showed that the usual $L_{z} / D_{v}$

Table 2. Classification of the 3 Typical Sky Conditions Using Hybrid Sky Parameter Lz/Dv-Gv/Ev

\begin{tabular}{|c|c|c|c|}
\hline Sky Conditions & Overcast & Partly Cloudy & Clear \\
\hline Proposed range & $\begin{array}{c}\mathrm{L}_{\mathrm{z}} / \mathrm{D}_{\mathrm{v}} \geq 0.3 \\
\& \\
\mathrm{G}_{\mathrm{v}} / \mathrm{E}_{\mathrm{v}} \leq 0.3\end{array}$ & $\begin{array}{c}0.17<\mathrm{L}_{\mathrm{z}} / \mathrm{D}_{\mathrm{v}}<0.3 \& \\
\mathrm{G}_{\mathrm{v}} / \mathrm{E}_{\mathrm{v}} \leq 0.3 \\
\text { or } \\
\mathrm{L}_{\mathrm{z}} / \mathrm{D}_{\mathrm{v}}>0.17 \& \\
0.3<\mathrm{G}_{\mathrm{v}} / \mathrm{E}_{\mathrm{v}}<0.5\end{array}$ & $\begin{array}{c}\mathrm{L}_{\mathrm{z}} / \mathrm{D}_{\mathrm{v}} \leq 0.17 \\
\text { or } \\
\mathrm{L}_{\mathrm{z}} / \mathrm{D}_{\mathrm{v}} \geq 0.17 \& \\
\mathrm{G}_{\mathrm{v}} / \mathrm{E}_{\mathrm{v}} \geq 0.5\end{array}$ \\
\hline
\end{tabular}


spay is greater than 0.38 for sky standard 1 and 0.33 to 0.38 for sky no. 3. It indicates that a value of the $\mathrm{L}_{\mathrm{z}} / \mathrm{D}_{\mathrm{v}}$ of 0.38 is the appropriate threshold. For partly cloudy conditions, sky standard 6 should be without direct-beam solar illuminance $\left(B_{v}\right)$. When there is no direct-beam component, it can be considered as sky standard 6 . The remaining 2 partly cloudy sky standards (sky nos. 7 and 8) can be classified by using the luminous turbidity $\left(T_{v}\right)$. The general formula for $T_{v}$ is given as follows [4]:

$\mathrm{T}_{\mathrm{v}}=\frac{\ln \left(\mathrm{E}_{\mathrm{v}} / \mathrm{B}_{\mathrm{v}}\right)}{\mathrm{a}_{\mathrm{v}} \mathrm{m}_{\mathrm{v}}} \ldots$

where $\mathrm{a}_{\mathrm{v}}=$ luminous ideal extinction $=\frac{1}{9.9+0.043 \mathrm{~m}_{\mathrm{v}}}$ [24],

$\mathrm{m}_{\mathrm{v}}=$ optical air mass $=\frac{1}{\sin \alpha_{\mathrm{S}}+0.50572\left(\alpha_{\mathrm{S}}+6.07995\right)^{-1.6364}}$

The typical $\mathrm{T}_{\mathrm{v}}$ values range between 5 and 12 for sky no. 8 and around 12 for sky no. 7 . The clear-cut $T_{v}$ value is 12 . The proposed criteria of $\mathrm{L}_{\mathrm{z}} / \mathrm{D}_{\mathrm{v}}, \mathrm{B}_{\mathrm{v}}$ and $\mathrm{T}_{\mathrm{v}}$ for the classification of the standard skies $1,3,6,7$ and 8 are summarized in (Fig. 4).

The same 68000 ten-minute sky luminance data were used to form and evaluate the classification of the 6 prevailing sky standards (sky nos. 1, 3, 6, 7, 8 and 13) in Hong Kong. Again, the RMSEs were computed for an assessment. (Fig. 5) displays (i) the frequency of occurrence and (ii) the RMSE results. As sky no. 13 has been selected to be the most representative clear skies from the CIE standard skies in Hong Kong, it obtains the largest frequency of occurrence of $36.5 \%$. This is not surprising, given that Hong Kong is currently infamous for its poor air quality and polluted conditions. Comparing with the best-fitting approach, the frequency of occurrence for sky no. 8 drops rapidly to $3.5 \%$ but sky no. 7 rises to over $16 \%$. For sky standards 1,3 and 6 , the frequency of occurrence varies slightly. Generally, Fig. 5 shows that the sky standards are quite evenly distributed in terms of the three typical sky types under such categorizations. The RMSEs range from $26.9 \%$ for sky standard 13 to $37.7 \%$ for sky standard 7 . The overall RMSE for the reduced set of the 6 prevailing sky standards in Hong Kong is $30 \%$.

\section{CONCLUSIONS}

An analysis of the CIE standard skies using various approaches against measured sky luminance data in Hong
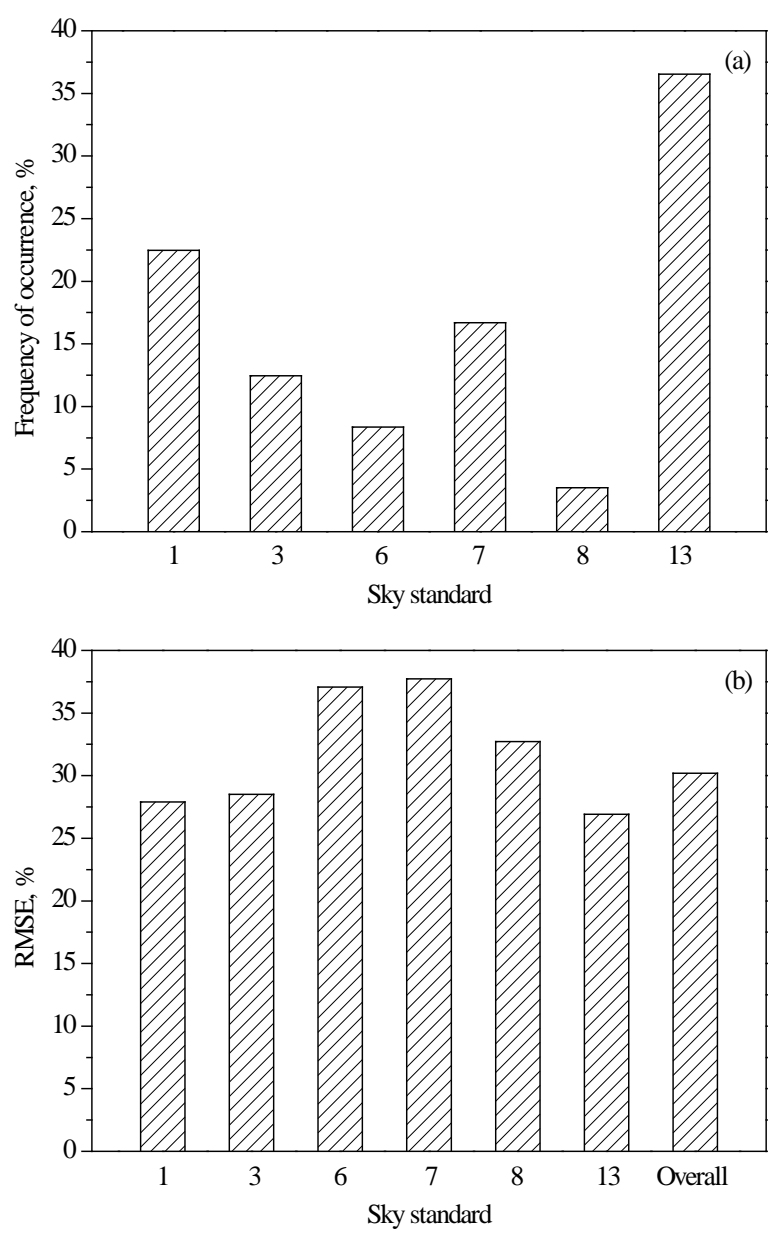

Fig. (5). (a) Frequency of occurrence, and (b) RMSEs of the luminance distribution of the prevalent skies to the $1,3,6,7,8$ and 13 skies using the proposed method.

Kong was conducted. Totally, 68000 ten-minute sky luminance data were used for the study. Based on the best-fitting approach, the appropriate sky types for subtropical Hong Kong were identified. The statistical analysis revealed that the 15 CIE standard skies are adequate to categorize the general sky conditions in Hong Kong. The RMSE value for the complete set of the 15 standard skies was $23.8 \%$. To simplify the study, a subset of 6 sky standards (sky nos. 1, 3, 6, 7, 8

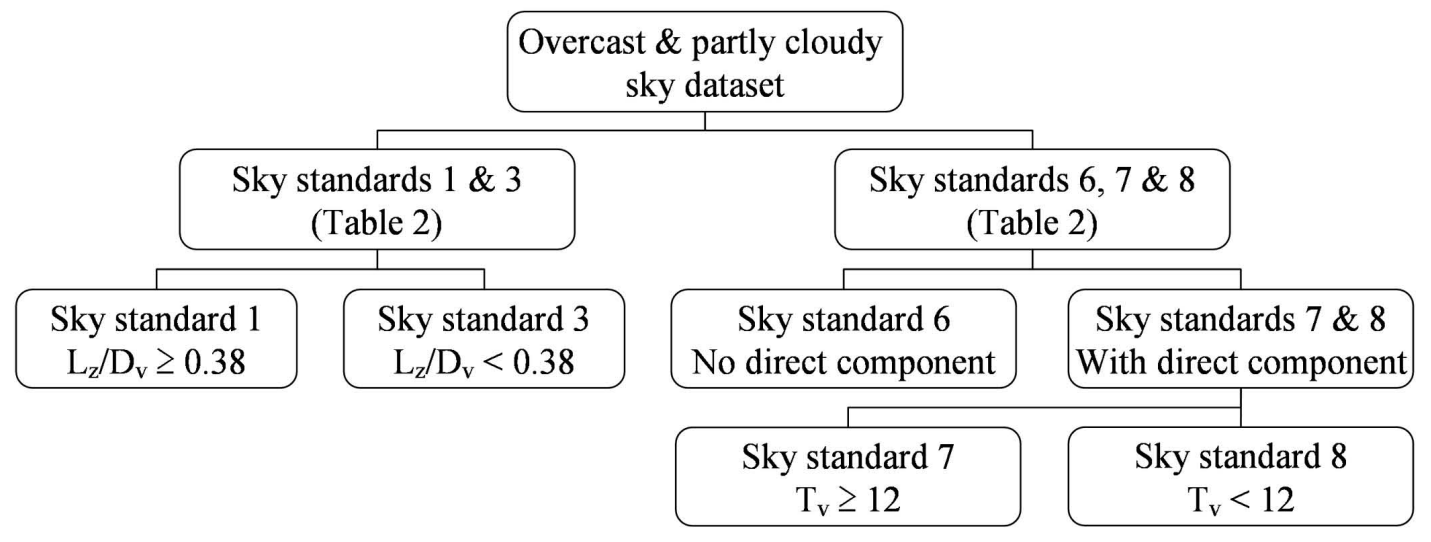

Fig. (4). The proposed criteria for the classification of the sky standards 1, 3, 6, 7 and 8 . 
and 13) representing the prevailing sky conditions in Hong Kong were selected. Using the hybrid-parameter $\mathrm{L}_{\mathrm{z}} / \mathrm{D}_{\mathrm{v}^{-}}$ $\mathrm{G}_{\mathrm{v}} / \mathrm{E}_{\mathrm{v}}$, all measured sky luminance distributions were initially interpreted as overcast, partly cloudy and clear sky types. A further analysis was carried out to identify sky standards 1 and 3 from the overcast sky dataset and sky standards 6,7 and 8 from the partly cloudy database with various climatic parameters including the ratio of zenith luminance to horizontal diffuse illuminance $\left(\mathrm{L}_{\mathrm{z}} / \mathrm{D}_{\mathrm{v}}\right)$ the direct-beam solar illuminance $\left(\mathrm{B}_{\mathrm{v}}\right)$, and luminous turbidity $\left(\mathrm{T}_{\mathrm{v}}\right)$. The overall RMSE was found to be $30 \%$ without further data rejection.

\section{ACKNOWLEDGEMENTS}

The work described in this paper was fully supported by a Public Policy Research Exercise from the Research Grants Council of the Hong Kong Special Administrative Region, China [Project No. 9050195 (CityU 1001-PPR-2)]. H. L. Tang is supported by a City University of Hong Kong studentship.

\section{REFERENCES}

[1] P. Moon and D.E. Spencer, "Illumination from a non uniform sky", Illuminating Engineering (N.Y.), vol. 37(10), pp. 707-726, 1942.

[2] P.J. Littlefair, "A comparison of sky luminance models with measured data from Garston, United Kingdom”, Solar Energy, vol. 53(4), pp. 315-322, 1994.

[3] R. Perez, R. Seals and J. Michalsky, "All-weather model for sky luminance distribution-preliminary configuration and validation", Solar Energy, vol. 50(3), pp. 235-245, 1993.

[4] R. Kittler, S. Darula and R. Perez, A set of standard skies, Bratislava, Polygrafia, 1998.

[5] CIE S 011/E. Spatial distribution of daylight - CIE standard general sky, Standard, CIE central bureau, Vienna, 2003.

[6] R. Kittler, R. Perez and S. Darula, "Sky classification respecting energy-efficient lighting, glare and control needs", Journal of Illuminating Engineering Society, vol. 26(1), pp. 57-68, 1997.

[7] D.H.W. Li and G.H.W. Cheung, "Average daylight factor for the 15 CIE standard skies”, Lighting Research and Technology, vol. 38(2), 137-152, 2006.

[8] P.R. Tregenza, "Standard skies for maritime climates", Lighting Research and Technology, vol. 32(3), pp. 97-106, 1999.

[9] D.H.W. Li, C.C.S. Lau and J.C. Lam, "A study of 15 sky luminance patterns against Hong Kong data”, Architectural Science Review, vol. 46(1), pp. 61-68, 2003.
[10] E. Ng, V. Cheng, A. Gadi, J. Mu, M. Lee and A. Gadi, "Defining standard skies for Hong Kong”, Building and Environment, vol. 42(2), pp. 866-876, 2007.

[11] M.T. Markou, H.D. Kambezidis, A. Bartzokas, B.D. Katsoulis and T. Muneer, "Sky type classification in Central England during winter”, Energy, vol. 30(9), pp. 1667-1674, 2005.

[12] A. Bartzokas, S. Darula, H.D. Kambezidis and R. Kittler, "Sky luminance distribution in Central Europe and the Mediterranean area during the winter period", Journal of Atmospheric and SolarTerrestrial Physics, vol. 65(1), pp. 113-119, 2003.

[13] A. Bartzokas, H.D. Kambezidis, S. Darula and R. Kittler, "Comparison between winter and summer sky-luminance distribution in Central Europe and in the Eastern Mediterranean”, Journal of Atmospheric and Solar-Terrestrial Physics, vol. 67(7), pp. 709-718, 2005.

[14] R. Kittler and S. Darula, "Parametric definition of the daylight climate”, Renewable Energy, vol. 26(2), pp. 177-187, 2002.

[15] D.H.W. Li, C.C.S. Lau and J.C. Lam, "Predicting daylight illuminance on inclined surfaces using sky luminance data", Energy, vol. 30(9), pp. 1649-1665, 2005.

[16] B.A. Lebaron, J.J. Michalsky and R. Perez, "A simple procedure for correcting shadowband data for all sky conditions", Solar Energy, vol. 44(5), pp. 249-256, 1990.

[17] R. Kittler, S. Darula and R. Perez, "Advantages of new sky standards: more realistic modeling of daylight conditions in energy and environmental studies", International Journal of Energy, Environment and Economics, vol. 8(1), pp. 65-71, 1999.

[18] P.J. Littlefair, "The luminance distribution of clear and quasi-clear skies", in Proceedings of the CIBSE National Lighting Conference, Cambridge, UK, 1994, pp. 267-283.

[19] R. Kittler, S. Darula and R. Perez, "A new generation of sky standards" in Proceedings of the LUX Europe Conference, Amsterdam, 1997, pp. 359-373.

[20] P.R. Tregenza, "Analysing sky luminance scans to obtain frequency distributions of CIE Standard General Skies”, Lighting Research and Technology, vol. 36(4), pp. 271-281, 2004.

[21] D.H.W. Li, C.C.S. Lau, J.C. Lam, "Standard skies classification using common climatic parameters", Journal of Solar Energy Engineering, vol. 126(3), pp. 957-964, 2004.

[22] D.H.W. Li, C.C.S. Lau, "An analysis of non-overcast sky luminance models against Hong Kong data”, Journal of Solar Energy Engineering, vol. 129(4), pp. 486-493, 2007.

[23] S. Darula and R. Kittler, "New trends in daylight theory based on the new ISO/CIE sky standard", Building Research Journal, vol. 52(3), pp. 181-197, 2004.

[24] M. Navvab, M. Karayel, E. Ne'eman and S. Selkovitz, “Analysis of atmospheric turbidity for daylight calculations”, Energy and Buildings, vol. 6(2-4), pp. 293-303, 1984.

[25] F. Kasten and A.T. Young, "Revised optical air mass tables and approximation formula”, Applied Optics, vol. 28(22), pp. 47354738, 1989. 